Supporting Information for:

\title{
Assessing PM2.5 Exposures with High Spatio-Temporal Resolution across the Continental United States
}

Qian Di $^{\dagger *}$, Itai Kloog ${ }^{\dagger+}$, Petros Koutrakis ${ }^{\dagger}$, Alexei Lyapustin ${ }^{\S}$, Yujie Wang ${ }^{\S}$, Joel Schwartz ${ }^{\dagger}$

${ }^{\dagger}$ Department of Environmental Health, Harvard T.H. Chan School of Public Heath, Boston, MA, 02115, USA

$\$$ Department of Geography and Environmental Development, Ben-Gurion University of the Negev, Beer Sheva, P.O.B. 653, Israel

${ }^{\S}$ GEST/UMBC, NASA Goddard Space Flight Center, Baltimore, MD, USA

Correspondence to: Qian Di, Phone: 814-777-8202, email: qiandi@mail.harvard.edu

This file includes:

Pages S1-S27

Methods

Tables S1 to S6

Figures S1 to S10

Reference 


\section{Methods}

\section{Study Area and Sub-regions}

Figure S1 displays the continental United States and $\mathrm{PM}_{2.5}$ monitoring site. The whole study area was divided into sub-region to calculate regional model. Sub-region was defined by US Census Division (Figure S2). States in each region are presented in Table S1.

\section{GEOS-Chem Outputs}

GEOS-Chem simulates different chemical components of $\mathrm{PM}_{2.5}$ rather than the total mass. Previous studies and GEOS-Chem manual have proposed several combinations of output fields

to simulate $\mathrm{PM}_{2.5} \cdot{ }^{1,2}$ By referring to previous studies and doing testing, we decided $\mathrm{PM}_{2.5}$ to be the sum of the output fields specified in Table S2. Logically, some of the output fields (e.g. PAN, PMN, PPM, and R4N2) are not components of $\mathrm{PM}_{2.5}$ and should be excluded from estimating $\mathrm{PM}_{2.5}$; but practically we found that including them improved correlation. Since our task is achieving better model performance, we finally decided to include those output fields in estimating $\mathrm{PM}_{2.5}$.

\section{Filling in Missing AOD Values}

Missing values in AOD was filled in by calibrating column aerosol mass from GEOS-Chem to satellite-based AOD using a neural network. We used meteorological variables, land-use terms and absorbing aerosol index as additional input layers of the neural network. The calibration work was done at daily basis. Since all input variables have no or almost no missing values, we can use the trained neural network to predict AOD for the whole study area, even at place with missing AOD values. Details of this work were specified somewhere else. ${ }^{3}$ 


\section{Regional Dummy Variable}

The process of creating regional dummy variables from major climate types is displayed in Figure S3. Data on climate types were obtained from somewhere else. ${ }^{4}$

\section{Convolution Layers}

A convolutional layer is computed by taking a convolutional kernel on an input layer over the entire area. The convolutional kernel function has constant parameters and can take many forms, such as mean, median or weighted averages. In a convolution layer, to obtain value of each cell, a kernel function is applied to neighboring cells and produces a scalar summary. Applying different kernel functions yields different convolutional layers.

Assuming we need to compute a convolutional layer for population density at monitoring sites. We chose the kernel function to be weighted average with weight proportional to the inverse of distance. For every site, we took distance-inversed weighted average of nearby population density. That is, for any monitor $k$, we have:

$$
Y_{k}=\frac{\sum_{i=1}^{n} w_{i k} y_{i}}{\sum_{i=1}^{n} w_{i k}}
$$

where $Y_{k}$ is the value of the convolutional layer at monitor $k, y_{i}$ is population density at grid cell $i ; w_{i k} \propto 1 / d_{i k}^{2} ; d_{i k}$ is the distance between grid cell $i$ and monitoring site $k$.

By changing other form of convolutional kernels, such as $w_{i k} \propto 1 / d_{i k}^{3}, w_{i k} \propto 1 / d_{i k}$, we could obtain other convolutional layers. 
The above example computes a convolutional layer over spatial neighborhood. It is also possible to have a convolutional layer over nearby days. For instance, a temporal convolutional layer takes moving average of predicted $\mathrm{PM}_{2.5}$ over the nearest $D$ days. The weight on each day is proportional to the day difference:

$$
Y_{k}=\frac{\sum_{i=-D}^{D} w_{i} y_{k}}{\sum_{i=-D}^{D} w_{i}}
$$

A convolutional layer is some form of aggregation of neighboring information for a variable. Different convolutional layers capture different levels of aggregation. Aggregation of nearby information makes it possible to capture autocorrelation. Multiple convolutional layers were put into a neural network as separate input layers. The neural network adjusts weights on each convolutional layer in learning process, in which various combinations of convolutional layers are explored until the optimized one is found. Combination of convolutional layers can simulate complex autocorrelation and possible interaction with other variables. More introductions about convolutional layers were specified somewhere else. ${ }^{5}$

\section{Neural Network}

Figure S4 presents the conceptual model of a neural network, in which interconnected "neurons" approximate some function. Input layer, hidden layer, and output layer all consist of neurons. Each neuron in the input layer represents one predictor, which could be AOD, OMI AAI, one land-use term, and others; neuron in the output layer represents predicted $\mathrm{PM}_{2.5}$, which is trained to $\mathrm{PM}_{2.5}$ monitoring data from AQS. Neural network works in the following way: each neuron takes weighted average from neurons in the previous layer and transforms the average by some nonlinear function (e.g. log-sigmoid or hyperbolic tangent sigmoid function). Nonlinear 
function accounts for possible interactions and nonlinearities. The same process applies to the neurons in the next layer until it reaches to the output layer. Initial weight for each neuron is randomly assigned and updated in learning process. In the learning process, a learning algorithm updates weights based on some rule to minimize the difference between output and target value. In this study, we used Levenberg-Marquardt optimization algorithm. ${ }^{6}$ More hidden layers result in better capacity to model nonlinearity but more computation time. One hidden layer was suggest to avoid overfitting. ${ }^{7}$ Our study involves complex and nonlinear atmospheric process. We used two hidden layer to have a compromise between model fit and computation time. Previous study suggested the number of neurons to be in the range of $(2 \sqrt{n}+u \sim 2 n+1)$, in which $n$ is number of input variables and $u$ is the number of output variables. ${ }^{8}$ We put 15 neurons in each hidden layer. To improve efficiency, we normalized input variables within the range of ($1,1)$ as preprocessing.

Convolutional layers were incorporated into a neural network just as ordinary input layers. Some convolutional layers are for predicted $\mathrm{PM}_{2.5}$, which requires us to fit a neural network in two stages. First, we fitted a neural network with AOD, OMI AAI, CTM outputs, $\mathrm{PM}_{2.5}$ vertical profile, meteorological variables, land-use terms and their convolutional layers, convolutional layers for nearby $\mathrm{PM}_{2.5}$ measurements, NDVI, surface reflectance, monthly/regional dummy variables. The target variable was AQS monitored $\mathrm{PM}_{2.5}$. After training, we obtained intermediated $\mathrm{PM}_{2.5}$ prediction from the neural network and computed both temporal and spatial convolutional layers for predicted $\mathrm{PM}_{2.5}$. We fitted the neural network again with above variables and convolutional layers for predicted $\mathrm{PM}_{2.5}$. The neural network yielded predicted $\mathrm{PM}_{2.5}$ with better model performance, which is the final predicted $\mathrm{PM}_{2.5}$. Correspondingly, we predicted 
$\mathrm{PM}_{2.5}$ at $1 \mathrm{~km} \times 1 \mathrm{~km}$ grid cells in a two-stage way. The two-stage modeling approach is displayed in Figure S5.

\section{Calculation $\mathbf{R}^{2}$}

We used total $\mathrm{R}^{2}$, spatial $\mathrm{R}^{2}$ and temporal $\mathrm{R}^{2}$ to quantify model performance. $\mathrm{R}^{2} \mathrm{~s}$ between cross-validated predicted $\mathrm{PM}_{2.5}$ and monitored $\mathrm{PM}_{2.5}$ were calculated based on description specified somewhere else. ${ }^{9}$ We fitted a regression of predicted $\mathrm{PM}_{2.5}$ on monitored $\mathrm{PM}_{2.5}$ and calculated $\mathrm{R}^{2}$ for this regression model, which was total $\mathrm{R}^{2}$. We fitted a regression of annual mean of predicted $\mathrm{PM}_{2.5}$ on annual mean of monitored $\mathrm{PM}_{2.5}$ and calculated $\mathrm{R}^{2}$ for this regression model, which was spatial $\mathrm{R}^{2}$. We fitted a regression of Delta Predicted on Delta Monitored and computed $\mathrm{R}^{2}$ for this model, which was temporal $\mathrm{R}^{2}$. Delta Predicted was the difference between predicted $\mathrm{PM}_{2.5}$ and annual mean of predicted $\mathrm{PM}_{2.5}$ at that location; Delta Monitor was defined in a similar way, the difference between monitored $\mathrm{PM}_{2.5}$ and annual mean of monitored $\mathrm{PM}_{2.5}$ at that location.

If restricting monitoring records to a time period, to a certain geographic area, or below certain concentrations, we can compute $\mathrm{R}^{2}$ by season (Table S3Table S4), by region (Table S4) or at low concentrations (Table S5).

\section{Model Comparison}

\subsection{Hybrid model}

Model with full set of variables performed better than models with the subset. To illustrate that, for each year, we fitted a model with GEOS-Chem, meteorological variables, and land-use terms as input variables. This was the benchmark model. We fitted the following three hybrid 
models by adding the following variables to the benchmark model: (1) convolution terms of land-use variables (Hybrid Model 1); (2) convolution terms of land-use variables, Aqua-AOD, and OMI AAI (Hybrid Model 2); (3) convolution terms of land-use variables, Aqua-AOD, TerraAOD and OMI AAI to the benchmark model (Hybrid Model 3). Compared with the Hybrid Model 3, the first two hybrid models were subset models. We calculated the cross-validated total $\mathrm{R}^{2}$. Figure $\mathrm{S} 6$ visualizes the improvement of model performance and indicates those improvements are of statistical significance. Hybrid Model 3, the one with full list of variables, improved the model performance more than models with subsets of variables. Cross-validated total $\mathrm{R}^{2}$ were reported to eliminate overfitting.

\subsection{Scaling factor}

Previous study used GEOS-Chem output to calculate the percentage of ground aerosol concentration in total column concentration as scaling factor and multiplied AOD by this scaling factor for calibration. ${ }^{10}$ We argue that the potential relationship between AOD and a scaling factor can be rather complex. We put the scaling factor input into neural network as a separate layer and let the neural network simulate possible complex relationships. To justify which approach is superior, we first fitted a benchmark model with Aqua/Terra-AOD, meteorological variables and land-use terms for each year. Then we included scaling factor either by multiplying with AOD (denoted as Model 1) or as a separate input layer (denoted as Model 2) and calculated the cross-validated total $\mathrm{R}^{2}$ s. Figure $\mathrm{S} 7$ indicates that including scaling factor as a separate layer significantly improves model fit even more.

\subsection{Convolutional Layers}


Convolutional layers account for temporal and spatial autocorrelation and improve model fit. To demonstrate that, we fitted a benchmark model with GEOS-Chem outputs, AOD, OMI AAI, meteorological variables and land-use terms. On the basis of that, we added either convolutional layers of nearby monitored $\mathrm{PM}_{2.5}$ and improved model with statistical significance. If further adding convolution terms of nearby predicted $\mathrm{PM}_{2.5}$, model fit can improve again with statistical significance (Figure S8).

\section{Model Diagnosis}

We evaluated the model performance at low concentrations (daily average $<35 \mu \mathrm{g} / \mathrm{m}^{3}$ ). Table S5 presents the cross-validated results when monitored $\mathrm{PM}_{2.5}$ below $35 \mu \mathrm{g} / \mathrm{m}^{3}$. Compared with Table 1, there was a slight increase in $\mathrm{R}^{2}$ and mean annual $\mathrm{R}^{2}$ increased 0.01 to 0.85 , which indicates good model performance at low concentrations at well. Figure S9 visualizes the difference between long-term average of predicted $\mathrm{PM}_{2.5}$ and monitored $\mathrm{PM}_{2.5}$. The difference is within $\pm 1 \mu \mathrm{g} / \mathrm{m}^{3}$ for most areas of the United States. Marine, Colorado, and Wisconsin witnessed slight overestimation of long-term averaged $\mathrm{PM}_{2.5}\left(<1 \mu \mathrm{g} / \mathrm{m}^{3}\right)$; while Montana has slight underestimation.

Besides, we also investigated the association between model performance with elevation and average $\mathrm{PM}_{2.5}$ level (Figure S10). We computed the overall model performance from 2000 2012 for each site and its correlation with in situ elevation and average $\mathrm{PM}_{2.5}$ in the same period.

There is a negative correlation between model performance and elevation, with $R^{2}=-0.2470$ and p-value $<0.0001$. Average $\mathrm{PM}_{2.5}$ positively associates with model performance, with $\mathrm{R}^{2}=0.2697$ and p-value $<0.0001$. 
Table S1. Census Bureau division

\begin{tabular}{|c|c|c|}
\hline Division & Name & States \\
\hline Division 1 & New England & $\begin{array}{c}\text { Connecticut, Maine, Massachusetts, New } \\
\text { Hampshire, Rhode Island, Vermont }\end{array}$ \\
\hline Division 2 & Middle Atlantic & New Jersey, New York, Pennsylvania \\
\hline Division 3 & East North Central & Indiana, Illinois, Michigan, Ohio, Wisconsin \\
\hline Division 4 & West North Central & $\begin{array}{c}\text { Iowa, Nebraska, Kansas, North Dakota, Minnesota, } \\
\text { South Dakota, Missouri }\end{array}$ \\
\hline Division 5 & South Atlantic & $\begin{array}{l}\text { Delaware, District of Columbia, Florida, Georgia, } \\
\text { Maryland, North Carolina, South Carolina, Virginia, } \\
\text { West Virginia }\end{array}$ \\
\hline Division 6 & East South Central & Alabama, Kentucky, Mississippi, Tennessee \\
\hline Division 7 & West South Central & Arkansas, Louisiana, Oklahoma, Texas \\
\hline Division 8 & Mountain & $\begin{array}{l}\text { Arizona, Colorado, Idaho, New Mexico, Montana, } \\
\text { Utah, Nevada, Wyoming }\end{array}$ \\
\hline Division 9 & Pacific & Alaska, California, Hawaii, Oregon, Washington \\
\hline
\end{tabular}

Note: contents are based on http://www2.census.gov/geo/pdfs/maps-data/maps 
Table S2. GEOS-Chem outputs used to calculate total $\mathbf{P M}_{2.5}$

\begin{tabular}{|c|c|c|}
\hline Name & Full Name & $\mathrm{g} / \mathrm{mol}$ \\
\hline PAN & Peroxyacetyl Nitrate & 121 \\
\hline PMN & Peroxymethacroyl Nitrate & 147 \\
\hline PPN & Lumped Peroxypropionyl Nitrate & 135 \\
\hline R4N2 & Lumped Alkyl Nitrate & 119 \\
\hline $\mathrm{SO} 4$ & Sulfate & 96 \\
\hline SO4s & Sulfate on surface of sea-salt aerosol & 96 \\
\hline NH4 & Ammonium & 18 \\
\hline NIT & Inorganic nitrates & 62 \\
\hline NITs & Inorganic nitrates on surface of sea-salt aerosol & 62 \\
\hline BCPI & Hydrophilic black carbon aerosol & 12 \\
\hline OCPI & Hydrophilic organic carbon aerosol & 12 \\
\hline BCPO & Hydrophobic black carbon aerosol & 12 \\
\hline OCPO & Hydrophobic organic carbon aerosol & 12 \\
\hline DST1 & Dust aerosol, Reff $=0.7$ microns & 29 \\
\hline DST2 & Dust aerosol, Reff $=1.4$ microns & 29 \\
\hline DST3 & Dust aerosol, Reff $=2.4$ microns & 29 \\
\hline SALA & $\begin{array}{l}\text { Accumulation mode sea salt aerosol }(\operatorname{Reff}=0.01- \\
0.5 \text { microns })\end{array}$ & 31.4 \\
\hline $\mathrm{BrNO} 3$ & Bromine nitrate & 142 \\
\hline MPN & Methyl peroxy nitrate & 93 \\
\hline
\end{tabular}




\begin{tabular}{|l|l|c|}
\hline ISOPN & Isoprene nitrate & 147 \\
\hline PROPNN & Propanone nitrate & 109 \\
\hline MMN & Nitrate from MACR + MVK & 149 \\
\hline
\end{tabular}


Table S3. Cross-validated $\mathbf{R}^{2}$ for seasons

\begin{tabular}{|c|c|c|c|c|c|c|c|c|c|c|}
\hline$\stackrel{\Xi}{\nexists}$ & 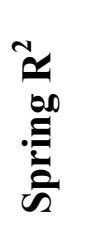 & $\sum_{\mathscr{a}}^{\underline{a}}$ & 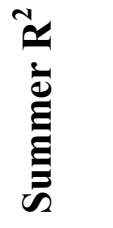 & $\sum_{\underline{\Omega}}^{\mathscr{\Omega}}$ & $\underset{\Xi}{\Xi}$ & $\sum_{\underline{\Omega}}^{\mathscr{\Omega}}$ & 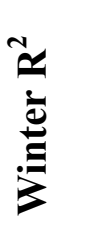 & $\sum_{\underline{\Omega}}^{\mathscr{\Omega}}$ & $\frac{\widetilde{Z}}{\widetilde{\Xi}}$ & $\sum_{\underline{\Lambda}}^{\mathbb{a}}$ \\
\hline 2000 & 0.85 & 2.60 & 0.89 & 2.81 & 0.88 & 3.48 & 0.79 & 4.62 & 0.86 & 3.35 \\
\hline 2001 & 0.85 & 2.85 & 0.92 & 2.70 & 0.78 & 4.25 & 0.82 & 3.48 & 0.84 & 3.58 \\
\hline 2002 & 0.87 & 2.12 & 0.90 & 3.22 & 0.89 & 2.79 & 0.83 & 3.50 & 0.88 & 2.99 \\
\hline 2003 & 0.89 & 2.34 & 0.92 & 2.54 & 0.87 & 2.89 & 0.82 & 3.50 & 0.88 & 2.80 \\
\hline 2004 & 0.87 & 2.30 & 0.93 & 2.18 & 0.89 & 2.64 & 0.83 & 3.48 & 0.88 & 2.69 \\
\hline 2005 & 0.87 & 2.34 & 0.92 & 2.58 & 0.89 & 2.88 & 0.81 & 3.47 & 0.88 & 2.94 \\
\hline 2006 & 0.88 & 2.17 & 0.91 & 2.46 & 0.86 & 2.58 & 0.80 & 3.72 & 0.86 & 2.77 \\
\hline 2007 & 0.86 & 2.87 & 0.89 & 2.79 & 0.86 & 2.83 & 0.83 & 3.24 & 0.87 & 2.95 \\
\hline 2008 & 0.83 & 2.13 & 0.88 & 2.68 & 0.85 & 2.54 & 0.80 & 3.45 & 0.85 & 2.64 \\
\hline 2009 & 0.85 & 1.96 & 0.87 & 1.92 & 0.78 & 2.89 & 0.81 & 3.52 & 0.82 & 2.73 \\
\hline 2010 & 0.80 & 2.60 & 0.83 & 2.50 & 0.78 & 2.72 & 0.80 & 3.53 & 0.81 & 2.85 \\
\hline 2011 & 0.81 & 2.24 & 0.84 & 2.59 & 0.79 & 2.56 & 0.77 & 3.65 & 0.81 & 2.83 \\
\hline 2012 & 0.72 & 2.60 & 0.72 & 2.93 & 0.75 & 3.70 & 0.74 & 3.29 & 0.74 & 3.15 \\
\hline Mean & 0.84 & 2.39 & 0.88 & 2.61 & 0.84 & 2.98 & 0.80 & 3.57 & 0.84 & 2.94 \\
\hline
\end{tabular}


Table S4. Cross-validated $\mathbf{R}^{2}$ for sub-regions

\begin{tabular}{|c|c|c|c|c|c|c|c|c|c|c|}
\hline$\stackrel{\Xi}{\beth}$ & 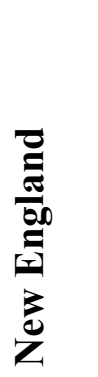 & 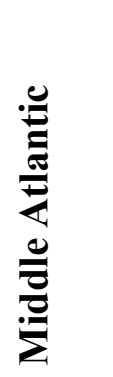 & 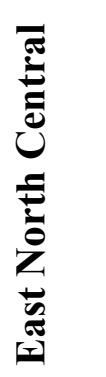 & 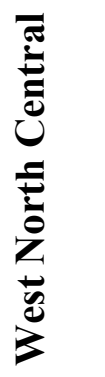 & 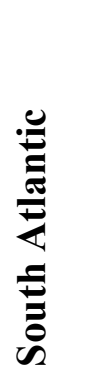 & 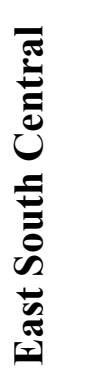 & 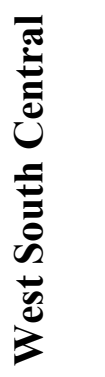 & & 氖 & 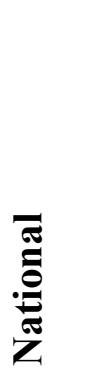 \\
\hline 2000 & 0.82 & 0.87 & 0.89 & 0.88 & 0.85 & 0.83 & 0.86 & 0.75 & 0.84 & 0.86 \\
\hline 2001 & 0.87 & 0.89 & 0.91 & 0.57 & 0.86 & 0.87 & 0.86 & 0.79 & 0.83 & 0.84 \\
\hline 2002 & 0.90 & 0.91 & 0.92 & 0.92 & 0.90 & 0.88 & 0.87 & 0.79 & 0.82 & 0.88 \\
\hline 2003 & 0.89 & 0.89 & 0.92 & 0.91 & 0.91 & 0.88 & 0.81 & 0.73 & 0.84 & 0.88 \\
\hline 2004 & 0.90 & 0.90 & 0.92 & 0.91 & 0.91 & 0.85 & 0.88 & 0.80 & 0.83 & 0.88 \\
\hline 2005 & 0.89 & 0.88 & 0.93 & 0.92 & 0.90 & 0.88 & 0.87 & 0.75 & 0.83 & 0.88 \\
\hline 2006 & 0.92 & 0.89 & 0.90 & 0.88 & 0.90 & 0.88 & 0.78 & 0.69 & 0.79 & 0.86 \\
\hline 2007 & 0.91 & 0.89 & 0.90 & 0.91 & 0.87 & 0.88 & 0.83 & 0.78 & 0.83 & 0.87 \\
\hline 2008 & 0.88 & 0.88 & 0.89 & 0.90 & 0.87 & 0.87 & 0.83 & 0.75 & 0.80 & 0.85 \\
\hline 2009 & 0.86 & 0.82 & 0.89 & 0.78 & 0.82 & 0.85 & 0.83 & 0.78 & 0.79 & 0.82 \\
\hline 2010 & 0.88 & 0.83 & 0.87 & 0.85 & 0.81 & 0.80 & 0.77 & 0.76 & 0.70 & 0.81 \\
\hline 2011 & 0.82 & 0.79 & 0.85 & 0.86 & 0.84 & 0.86 & 0.76 & 0.76 & 0.78 & 0.81 \\
\hline 2012 & 0.78 & 0.75 & 0.80 & 0.79 & 0.78 & 0.81 & 0.73 & 0.70 & 0.71 & 0.74 \\
\hline Mean & 0.87 & 0.86 & 0.89 & 0.85 & 0.86 & 0.86 & 0.82 & 0.76 & 0.80 & 0.84 \\
\hline
\end{tabular}


Table S5. Cross-validated $\mathbf{R}^{2}$ for $\mathbf{P M}_{2.5}<35 \mu \mathrm{g} / \mathrm{m}^{3}$

\begin{tabular}{|c|c|c|c|c|c|c|c|c|}
\hline 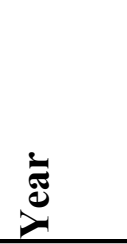 & $\frac{\widetilde{\sigma}}{\tilde{\sigma}}$ & $\sum_{\Sigma}^{\infty}$ & 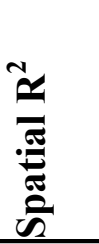 & $\sum_{\Sigma}^{\sqrt[1]{2}}$ & 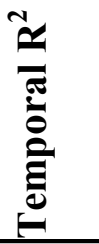 & $\sum^{\underline{a}}$ & 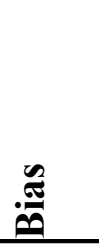 & $\frac{\check{0}}{\frac{0}{0}}$ \\
\hline 2000 & 0.86 & 2.71 & 0.85 & 1.30 & 0.85 & 2.44 & 0.69 & 0.96 \\
\hline 2001 & 0.87 & 2.59 & 0.87 & 1.17 & 0.86 & 2.37 & 0.66 & 0.96 \\
\hline 2002 & 0.87 & 2.43 & 0.86 & 1.12 & 0.87 & 2.21 & 0.66 & 0.95 \\
\hline 2003 & 0.87 & 2.36 & 0.86 & 1.12 & 0.88 & 2.14 & 0.58 & 0.96 \\
\hline 2004 & 0.88 & 2.28 & 0.81 & 1.27 & 0.89 & 2.05 & 0.50 & 0.97 \\
\hline 2005 & 0.88 & 2.49 & 0.83 & 1.32 & 0.88 & 2.23 & 0.56 & 0.97 \\
\hline 2006 & 0.87 & 2.38 & 0.82 & 1.21 & 0.87 & 2.13 & 0.49 & 0.97 \\
\hline 2007 & 0.87 & 2.44 & 0.84 & 1.16 & 0.87 & 2.21 & 0.57 & 0.96 \\
\hline 2008 & 0.86 & 2.24 & 0.78 & 1.16 & 0.87 & 2.01 & 0.44 & 0.97 \\
\hline 2009 & 0.84 & 2.22 & 0.81 & 1.01 & 0.84 & 2.04 & 0.42 & 0.97 \\
\hline 2010 & 0.82 & 2.53 & 0.82 & 1.13 & 0.82 & 2.27 & 0.62 & 0.96 \\
\hline 2011 & 0.82 & 2.50 & 0.81 & 1.06 & 0.82 & 2.27 & 0.61 & 0.96 \\
\hline 2012 & 0.76 & 2.56 & 0.74 & 1.08 & 0.76 & 2.33 & 0.64 & 0.96 \\
\hline Mean & 0.85 & 2.44 & 0.82 & 1.16 & 0.85 & 2.21 & 0.57 & 0.96 \\
\hline
\end{tabular}


Table S6. Model performance compared with existing regional studies

\begin{tabular}{|c|c|c|c|c|c|c|}
\hline \multirow{3}{*}{$\begin{array}{l}\text { Northeastern } \\
\text { United States }\end{array}$} & \multirow{3}{*}{$\begin{array}{l}\text { Hybrid } \\
\text { Model }\end{array}$} & \multirow{3}{*}{$\begin{array}{l}\text { Existing } \\
\text { Study }^{\text {a }}\end{array}$} & \multirow{3}{*}{$\begin{array}{l}\text { Existing } \\
\text { Study }^{\text {b }}\end{array}$} & \multirow{3}{*}{$\begin{array}{l}\text { Southeastern } \\
\text { United } \\
\text { States }\end{array}$} & \multirow{3}{*}{$\begin{array}{l}\text { Hybrid } \\
\text { Model }\end{array}$} & \multirow{3}{*}{$\begin{array}{l}\text { Existing } \\
\text { Study }^{\mathrm{c}}\end{array}$} \\
\hline & & & & & & \\
\hline & & & & & & \\
\hline 2000 & 0.82 & 0.73 & & 2000 & 0.85 & N/A \\
\hline 2001 & 0.87 & 0.84 & & 2001 & 0.86 & N/A \\
\hline 2002 & 0.90 & 0.85 & & 2002 & 0.90 & N/A \\
\hline 2003 & 0.89 & 0.85 & 0.89 & 2003 & 0.91 & $0.72 \sim 0.83$ \\
\hline 2004 & 0.90 & 0.85 & 0.89 & 2004 & 0.91 & $0.74 \sim 0.80$ \\
\hline 2005 & 0.89 & 0.84 & 0.88 & 2005 & 0.90 & $0.75 \sim 0.83$ \\
\hline 2006 & 0.92 & 0.86 & 0.89 & 2006 & 0.90 & $0.74 \sim 0.84$ \\
\hline 2007 & 0.91 & 0.90 & 0.90 & 2007 & 0.87 & $0.70 \sim 0.85$ \\
\hline 2008 & 0.88 & 0.81 & 0.88 & 2008 & 0.87 & $0.69 \sim 0.78$ \\
\hline 2009 & 0.86 & N/A & 0.86 & 2009 & 0.82 & $0.66 \sim 0.78$ \\
\hline 2010 & 0.88 & N/A & 0.90 & 2010 & 0.81 & $0.65 \sim 0.80$ \\
\hline 2011 & 0.82 & N/A & 0.82 & 2011 & 0.84 & $0.63 \sim 0.79$ \\
\hline 2012 & 0.78 & N/A & & 2012 & 0.78 & N/A \\
\hline
\end{tabular}

a. Results cited from Table 1, Kloog et al. (2011) $;{ }^{9}$ b. Results cited from Table 1, Kloog et al. (2014); ${ }^{11}$ c. Results cited from Table 2, Lee et al. (2015). ${ }^{12}$ 


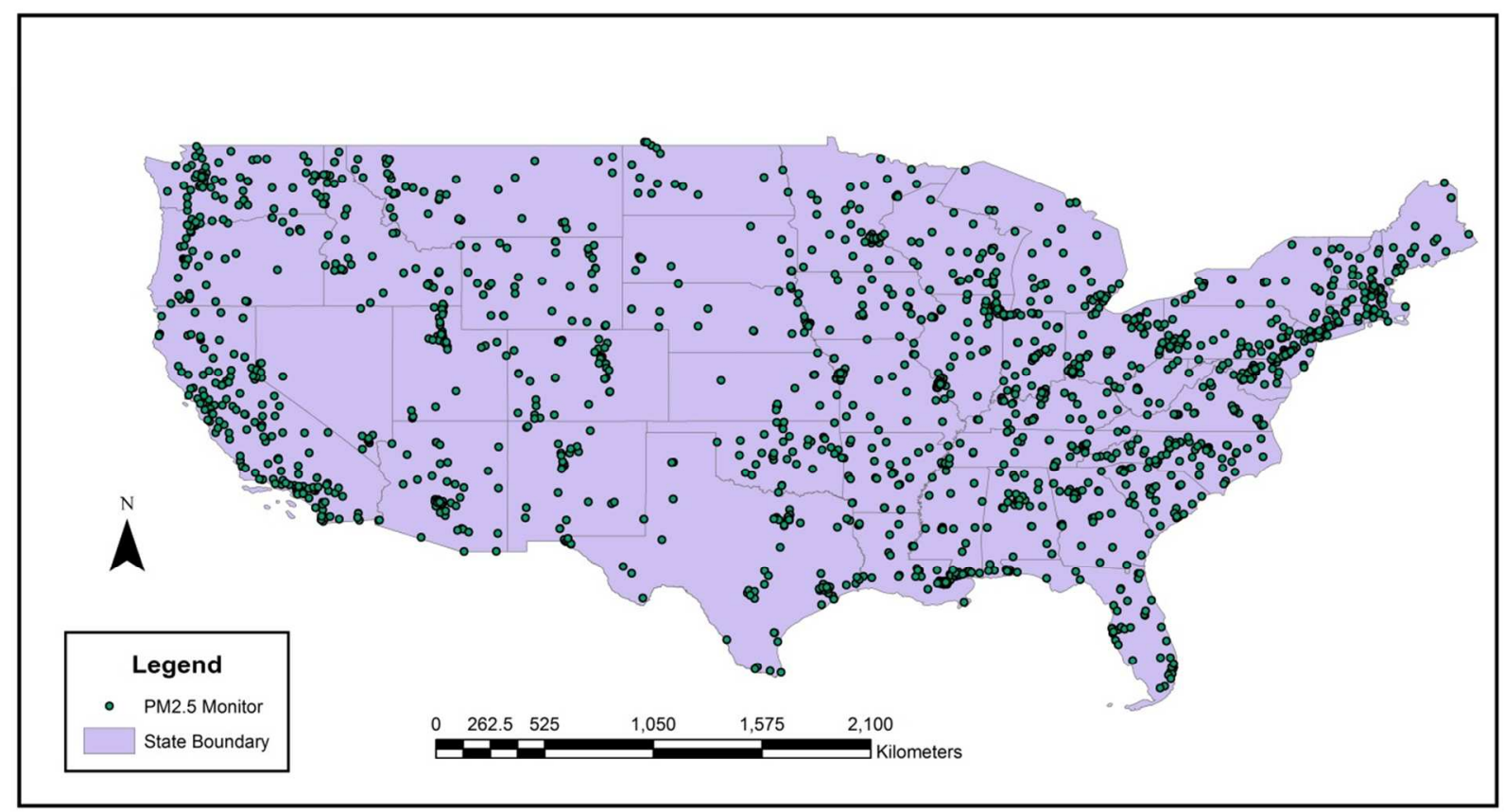

Figure S1. Map of the study area with $\mathbf{P M}_{2.5}$ monitors 


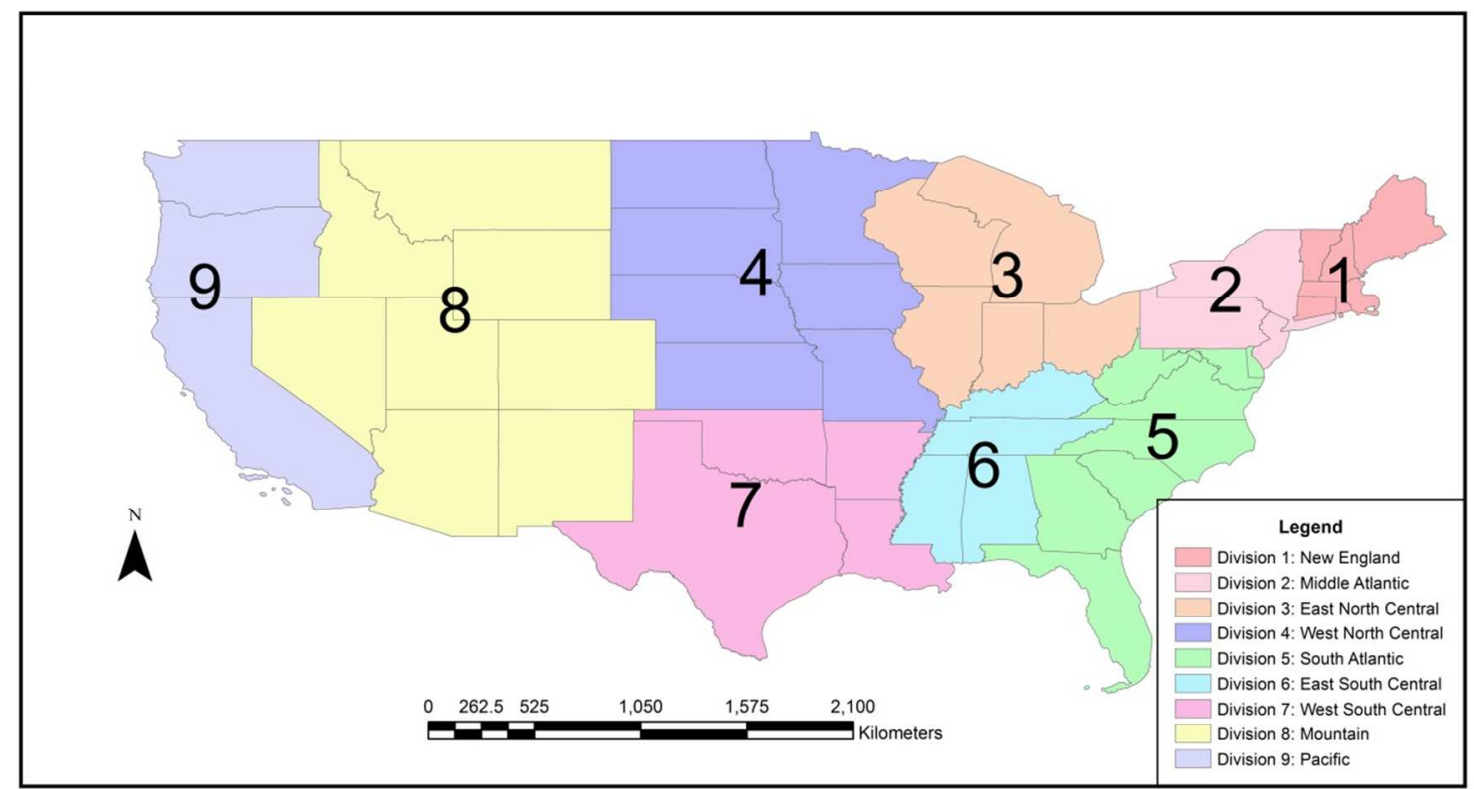

Figure S2. U.S. Census division

Hawaii, Alaska and other overseas territories are not included in the study area and not shown in the figure. 


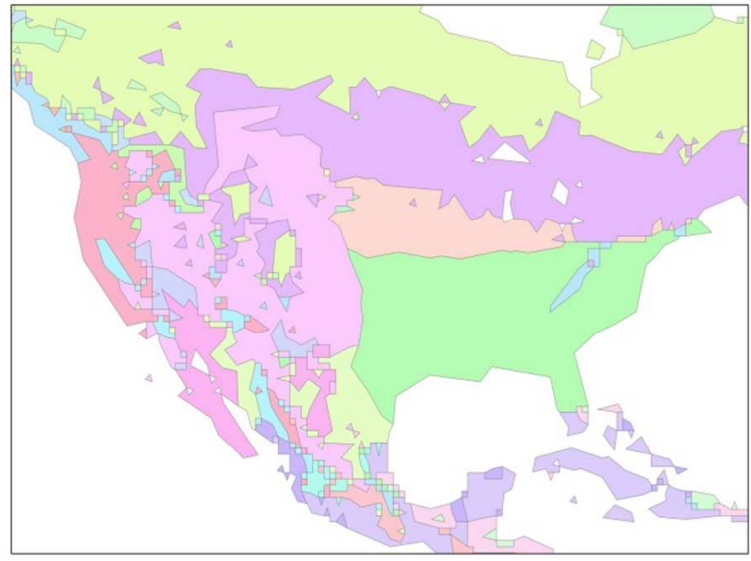

(a)

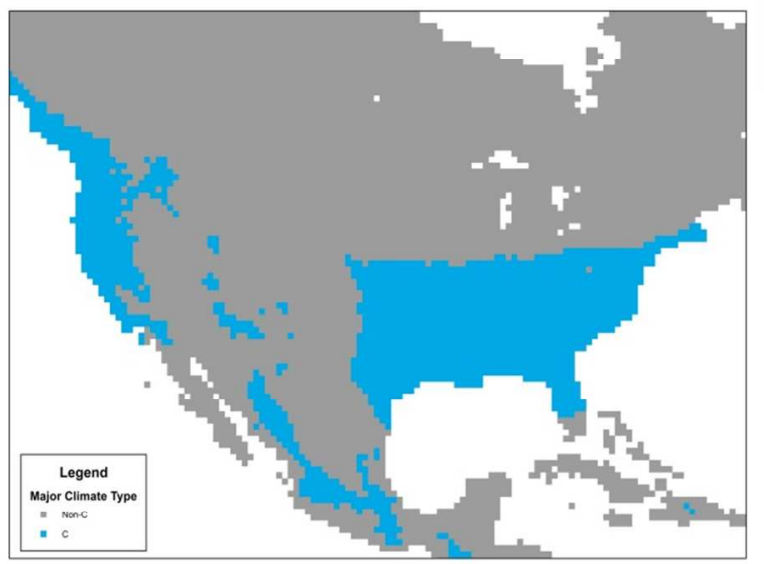

(c)

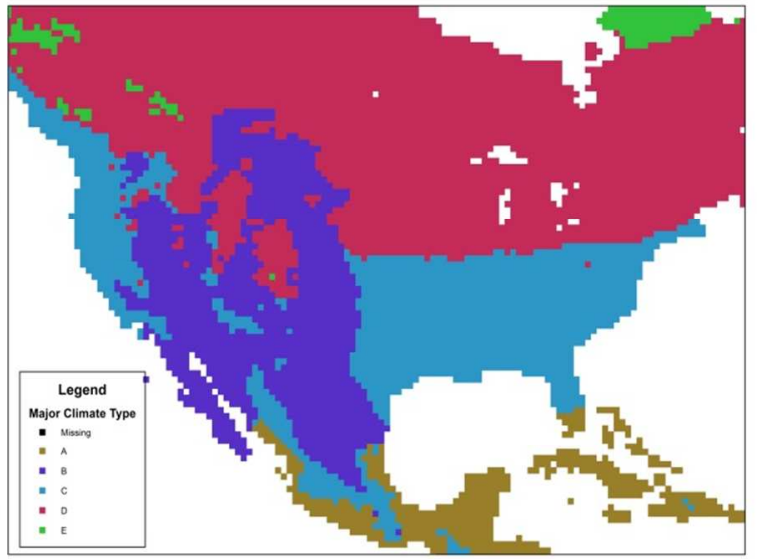

(b)

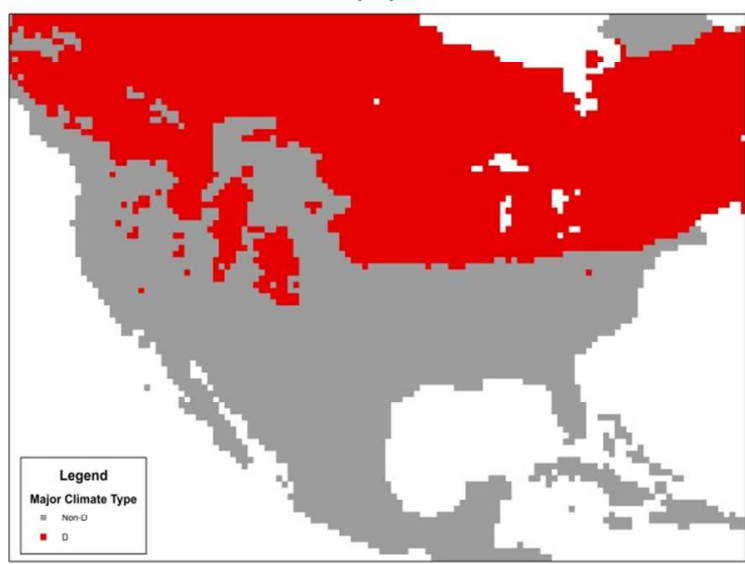

(d)

\section{Figure S3. Regional dummy from major climate types}

The original climate classification is presented in (Figure (a)). We aggregated minor climate types to five major types: A (equatorial climates), B (arid climates), C (warm temperate climates), D (snow climates) and E (Polar climates) in (Figure (b)). ${ }^{4}$ For each major climate type, we created a regional dummy. Take major climate type $\mathrm{C}$ as an example, we assigned regional dummy to be 1 for all grid cells covered by climate type $\mathrm{C}$ (blue areas in Figure (c)) and 0 otherwise (grey areas in Figure (c)). The same also held for climate type D (Figure (d)) and other types. 


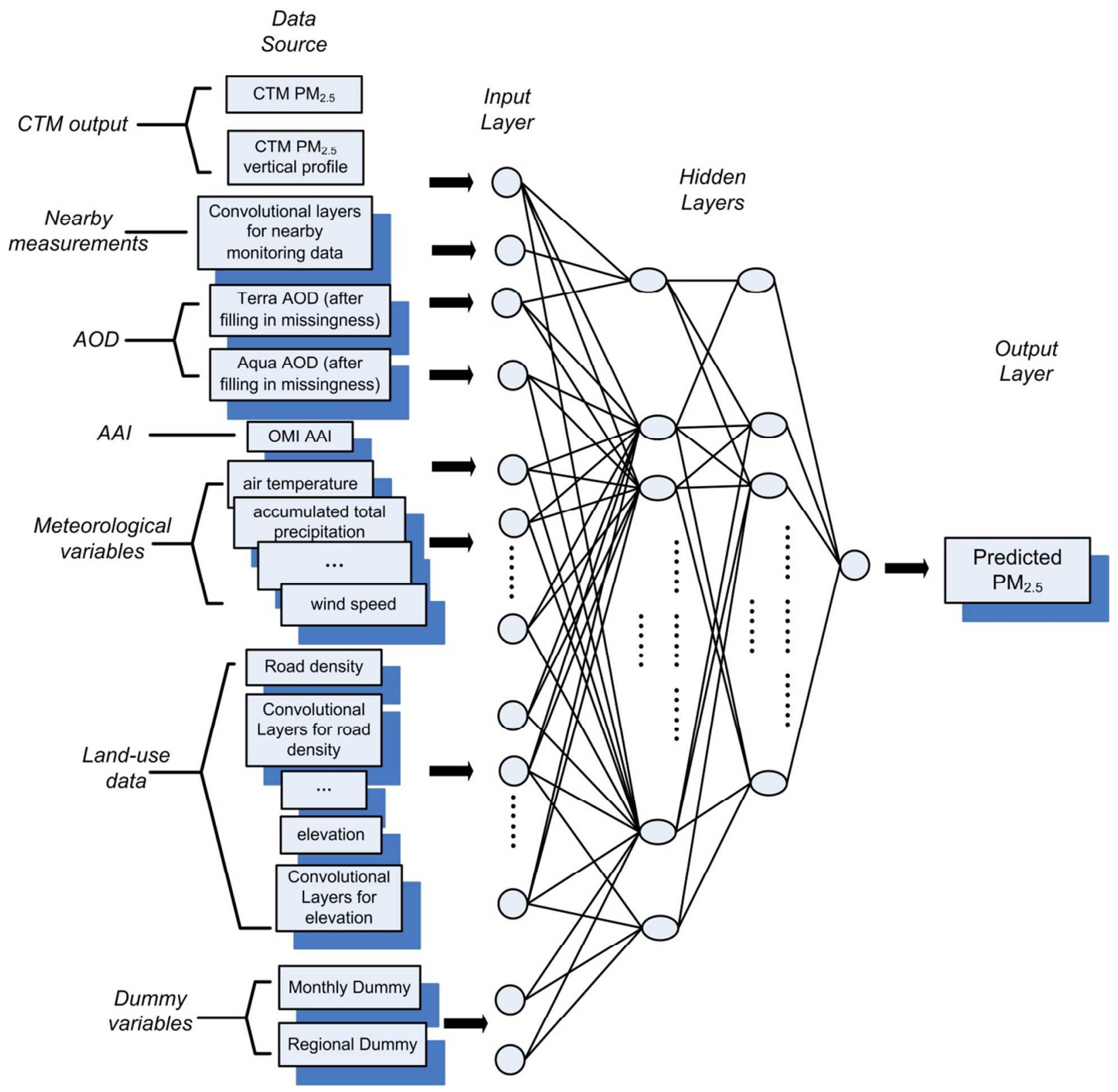

Figure S4. Conceptual model of neural model 


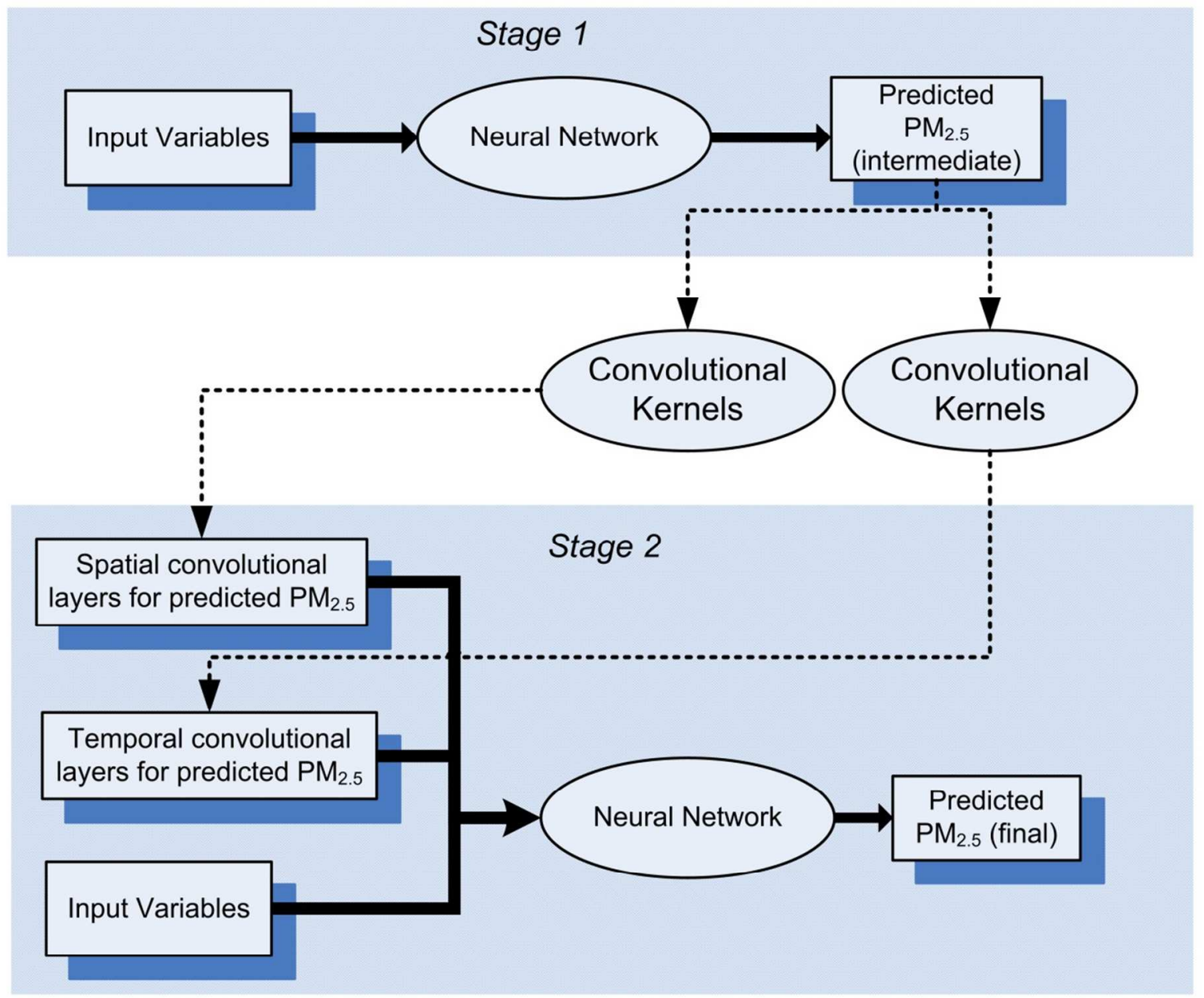

Figure S5. Flowchart of two-stage modeling approach

Note: We used Input Variables to stand for all input variables, which include AOD, OMI AAI, CTM outputs, $\mathrm{PM}_{2.5}$ vertical profile, meteorological variables, land-use terms and their convolutional layers, convolutional layers for nearby $\mathrm{PM}_{2.5}$ measurements, NDVI, surface reflectance, and monthly/regional dummy variables. 


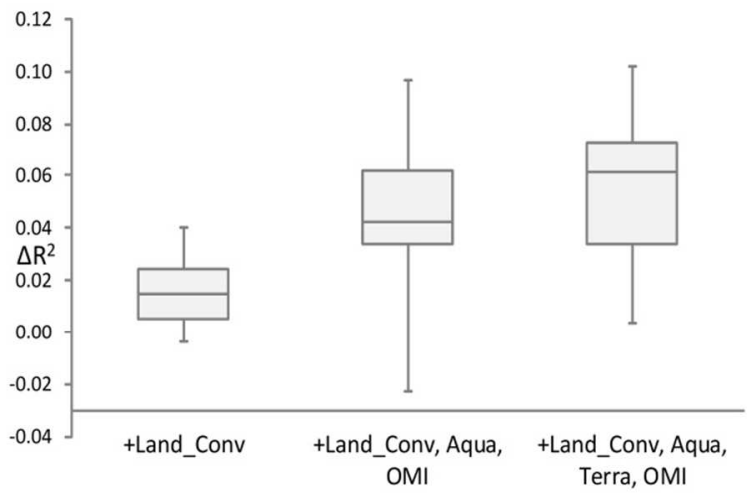

Figure S6. Improvement of model fit after adding extra variables

After adding convolution terms of land-use variables to the benchmark model (Hybrid Model 1), model fit improved $(\mathrm{p}<0.0001$; one-sided paired Wilcoxon signed rank test, the same below). If further adding Aqua-AOD and OMI AAI (Hybrid Model 2), model fit improved ( $\mathrm{p}=0.0133$ ).

Further adding Terra-AOD also improved model fit $(\mathrm{p}=0.0054)$. 


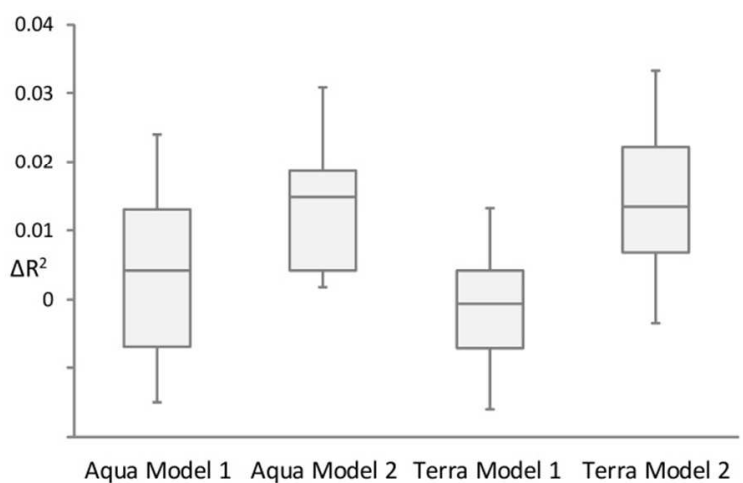

\section{Figure S7. Improvement of model fit after adding scaling factor}

Input layers of benchmark model: AOD, meteorological variables and land-use terms; input layers of Model 1: AOD*scaling factor, meteorological variables and land-use terms; input layers of Model 2: AOD, scaling factor, meteorological variables and land-use terms. Y-axis is the improvement of $\mathrm{R}^{2}$ compared with benchmark model. For Model $1, \mathrm{R}^{2}$ increased by 0.0034 $(p=0.0020$, one-sided paired Wilcoxon signed rank test, the same below) and $-0.0008(p=0.6890)$

for Aqua and Terra respectively; for Model $2, \mathrm{R}^{2}$ increased by $0.0139(\mathrm{p}=0.0010)$ and 0.0149 $(p=0.0021)$ for Aqua and Terra respectively. Model 2 demonstrated further improvement than Model 1 ( $p=0.0020$ for Aqua and $p=0.0002$ for Terra). 


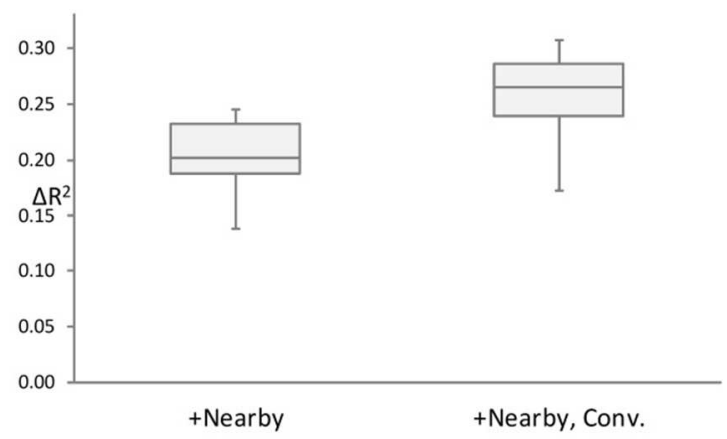

Figure S8. Improvement of model fit after adding convolutional layers

Input layers of benchmark model: GEOS-Chem outputs, Aqua-AOD, Terra-AOD, OMI AAI, meteorological variables and land-use terms. After adding nearby monitoring data as a convolutional layer into the model, $\mathrm{R}^{2}$ increased by $0.2043(\mathrm{p}=0.0001)$. If further adding convolutional layer of predicted $\mathrm{PM}_{2.5}, \mathrm{R}^{2}$ increased by $0.0517(\mathrm{p}=0.0001)$. 


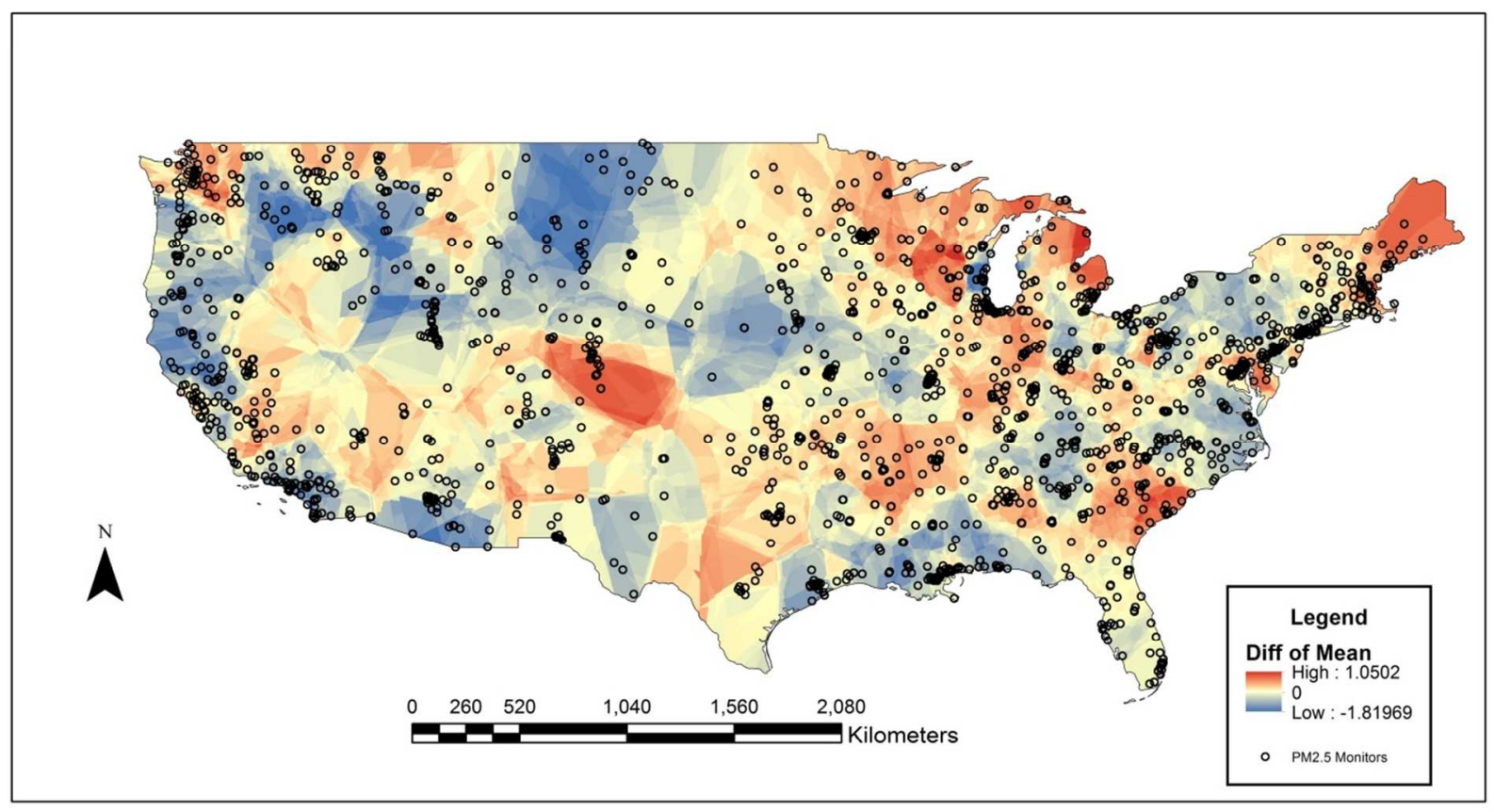

Figure S9. Difference between predicted long-term average and monitored long-term average

Note: We took the difference between long-term average of predicted $\mathrm{PM}_{2.5}$ and long-term average of monitored $\mathrm{PM}_{2.5}$ (from 2000 to 2012) at each monitoring site and interpolated it to places without monitors. After visualization, red color means predicted $\mathrm{PM}_{2.5}>$ monitored $\mathrm{PM}_{2.5}$ in the long-term (overestimation) and blue color means the opposite. Light yellow indicates prediction and monitoring values are close. Unit in this map is $\mu \mathrm{g} / \mathrm{m}^{3}$. 


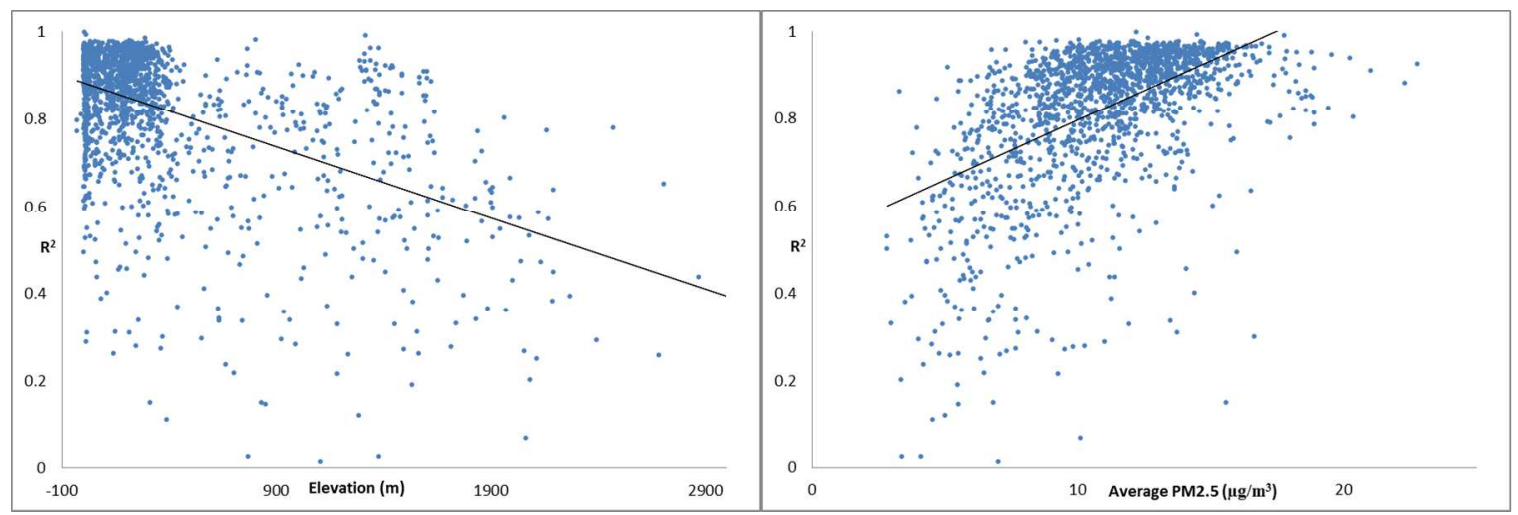

Figure S10. Model performance versus elevation and annual average $\mathbf{P M}_{2.5}$ 


\section{REFERENCES}

(1) Isakov, V.; Touma, J.; Touma, J.; Khlystov, A.; Sattler, M.; Devanathan, S.; Devanathan, S.; Engel-Cox, J.; Weber, S.; McFarland, M.; et al. Estimating Fine Particulate Matter Component Concentrations and Size Distributions Using Satellite-Retrieved Fractional Aerosol Optical Depth: Part 2-A Case Study. J. Air Waste Manage. 2007, 57, 1360-1369.

(2) Liu, Y. Mapping annual mean ground-level $\mathrm{PM}_{2.5}$ concentrations using Multiangle Imaging Spectroradiometer aerosol optical thickness over the contiguous United States. $J$. Geophys. Res. 2004, 109.

(3) Di, Q.; Schwartz, J., Using Chemical Transport Model to Fill in the Missingness of Satellite-Based AOD. Atmospheric Environment 2015 (in review).

(4) Kottek, M.; Grieser, J.; Beck, C.; Rudolf, B.; Rubel, F., World map of the Köppen-Geiger climate classification updated. Meteorologische Zeitschrift 2006, 15, (3), 259-263.

(5) LeCun, Y.; Ranzato, M. Deep learning tutorial. Tutorials in International Conference on Machine Learning (ICML'13), June $16^{\text {th }}, 2013$.

(6) Moré, J. J. The Levenberg-Marquardt algorithm: implementation and theory. Num. Anal. Springer Berlin Heidelberg 1978, 630, 105-116.

(7) Schalkoff, R. J. Artificial neural networks. McGraw-Hill: New York, 1997.

(8) Fletcher, D.; Goss, E. Forecasting with neural networks. Inform. Manage. 1993, 24, 159167.

(9) Kloog, I.; Koutrakis, P.; Coull, B. A.; Lee, H. J.; Schwartz, J. Assessing temporally and spatially resolved $\mathrm{PM}_{2.5}$ exposures for epidemiological studies using satellite aerosol optical depth measurements. Atmos. Environ. 2011, 45, 6267-6275.

(10) van Donkelaar, A.; Martin, R. V.; Park, R. J. Estimating ground-level PM 2.5 using aerosol optical depth determined from satellite remote sensing. J. Geophys. Res. 2006, 111.

(11) Kloog, I.; Chudnovsky, A. A.; Just, A. C.; Nordio, F.; Koutrakis, P.; Coull, B. A.; Lyapustin, A.; Wang, Y.; Schwartz, J. A new hybrid spatio-temporal model for estimating daily multi-year PM 2.5 concentrations across northeastern USA using high resolution aerosol optical depth data. Atmos. Environ. 2014, 95, 581-590. 
(12) Lee, M.; Kloog, I.; Chudnovsky, A.; Lyapustin, A.; Wang, Y.; Melly, S.; Coull, B.; Koutrakis, P.; Schwartz, J. Spatiotemporal prediction of fine particulate matter using highresolution satellite images in the Southeastern US 2003-2011. J. Expo. Sci. Env. Epid. 2015. 